\title{
Infrared thermography to inline monitoring of glass fibres composites under impact and quasi-static bending tests
}

\author{
by S. Boccardi*, G.M. Carlomagno*, C. Meola*, P. Russo**, G. Simeoli**

\begin{abstract}
* Department of Industrial Engineering - Aerospace Division, University of Naples Federico II, Naples, Italy, simone.boccardi@unina.it,carmagno@unina.it, carmeola@unina.it

${ }^{* *}$ Institute for Polymers, Composites and Biomaterials, National Council of Research, Pozzuoli (Na), Italy, pietro.russo@unina.it,giorgio.simeoli@unina.it
\end{abstract}

\begin{abstract}
This work would bear witness for the benefits of using infrared thermography during characterization of composite materials. Specimens made of glass fibres embedded in either a thermoset, or a thermoplastic, matrix are considered and subjected to mechanical tests: impact and quasi-static bending. Impact tests are performed with a modified Charpy pendulum, while quasi-static bending with a standard three point testing configuration setup. An infrared camera views the specimen surface opposite to the load application and acquires sequences of thermal images at a given frame rate. The acquired sequences are later post-processed to get information about damage initiation and propagation.
\end{abstract}

\section{Introduction}

Composite materials are being used for many years and are ever more increasingly employed in many industrial applications for the transport industry, the building sector as well for the production of many daily life objects. Their success is mainly due to their high strength-to-weight ratio, easy formability and relatively low costs.

Initially, the development of composites was strongly driven by aircraft requirements, mainly leading to the use of thermoset polymers as matrix because of their low density (low weight); however, their main weakness is their low interlaminar strength, which makes them susceptible to delamination under impact load. The introduction of composites in other transport sectors - like the automotive, naval and railways - for which the lightness is not an imperative as in airplanes, has driven the research interest towards the use of thermoplastic matrices. The latter offer some advantages over thermosets in terms of costs and disposal after use since a thermoplastic matrix can be re-melt and re-used [1].

However, the introduction of a new material requires thorough knowledge of its properties and performance inservice, which is a long way on requiring many tests and related effective instrumentation [2].

The intention of the present work is to show the benefits provided by infrared thermography in the characterization of composite materials. The investigation is driven towards composites made of glass fibres embedded in either a thermoset, or a thermoplastic, matrix and subjected to both impact, and quasi-static bending, tests. Impact tests are performed with a modified Charpy pendulum which, unlike the commonly used falling dart machines, allows enough room for positioning of the infrared camera to view the specimen surface opposite to the impact [3]; sequences of thermal images are collected with a cooled detector camera. A microbolometric handheld camera is used to view and record images during quasi-static bending with a standard three-point bending configuration set-up [4]. The obtained results show the usefulness of infrared thermography in both types of tests.

\section{Materials and testing procedures}

Three varieties of specimens are prepared by considering glass fibres as reinforcement of three different matrices: epoxy resin, pure polypropylene (PP) and polypropylene with the addition of a $2 \%$ of polypropylene grafted maleic anhydride (PP-g-MA) coupling agent. Glass/epoxy specimens, also named GFRP, are translucent allowing visibility, to the naked eye, of imperfections for a direct comparison with results supplied by infrared thermography. The second type of specimens are named PPG, while the last involving the compatibilizer are named PGC2. Each specimen is subjected to two types of tests: impact and quasi-static bending, both monitored with an infrared camera. Specimens are $3 \mathrm{~mm}$ thick and of different dimensions as required by the two different testing configurations.

Impact tests are performed with a modified Charpy pendulum with a hemispherical shaped nose hammer, 12.7 $\mathrm{mm}$ in diameter. each specimen is placed inside a special fixture which includes two large plates, each having a window $12.5 \mathrm{~cm} \times 7.5 \mathrm{~cm}$ to allow for the contact with the hammer from one side and optical view (by the infrared camera) from the other one. A small sample of the same material is positioned over one corner of the viewed window and is used as reference to correct the camera noise. The impact energy is set by suitably adjusting the falling height of the Charpy arm.

Quasi-static bending tests are carried out with a standard three point testing configuration setup. The specimen (130 mm long and $30 \mathrm{~mm}$ wide) is horizontally laid over two supports at a mutual distance of $50 \mathrm{~mm}$ and loaded downwards in the middle at a speed of $5 \mathrm{~mm} / \mathrm{min}$ with a $5 \mathrm{~mm}$ radius loading nose. The infrared camera is positioned so as to contemporaneously view each specimen from its bottom surface and thickness. 


\section{Analysis of results}

The acquired sequences of images are post-processed by using the ResearchIR software supplied with the infrared camera and routines specifically developed in the Matlab environment. Of course, a different treatment is reserved to images acquired during the two different tests since images taken during impact refer to an almost static scene, while those taken during quasi-static testing depict the deformation (bending) of the specimen from its flat initial configuration.

Starting from impact tests, the influence of the ambient temperature is reduced by subtracting an image taken at environmental conditions, before starting of impact tests, from all the subsequent images of the sequence; thus a sequence of $\Delta T$ images is created. As an example, for each of the three specimens, a $\Delta T$ image taken at $t=0.012 \mathrm{~s}$ during impact at $11.7 \mathrm{~J}$ is shown in figure 1 . As can be seen, despite the same impact energy, $\Delta T$ images are completely different. The first image of the PPG specimen is characterized by dark ray beams, which indicates wrapping of the material under the impactor pushing force; the lighter central zone shows a mild temperature rise to about $0,5 \mathrm{~K}$. The addition of the coupling agent improves fibre-matrix interface strength and makes the material more brittle; as a direct consequence, the PGC2 specimen is unable to undergo large deformations and breaks under impact. The temperature rise is much higher to up 30 $\mathrm{K}$. At last, the $\Delta T$ image of the GFRP specimen is characterized by hot spots mostly over horizontal fibres with an increase of temperature over $30 \mathrm{~K}$.

Two thermal images for each specimen taken during quasi-static bending are shown in figure 2. The first one refers to the appearance of the first hot spot accounting for initiation of failure and the other one for complete collapse. For all specimens, it is possible to notice the appearance of the first hot spot on the top layer (in contact with the pushing nose), meaning that failure arises in compression, but at different times: GFRP at $56.32 \mathrm{~s}$, PPG at $21.87 \mathrm{~s}$ and PGC2 at $25.70 \mathrm{~s}$. Also complete collapse occurs at different times and in different ways, i.e. in a zigzag fashion for the PPG and through a net straight line for the PGC2.

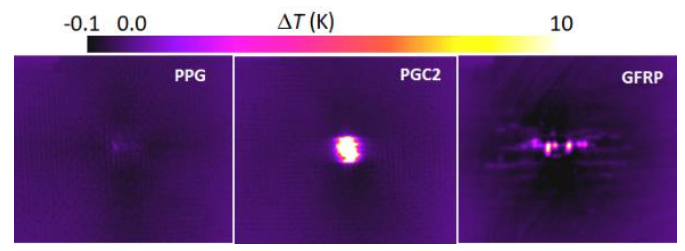

Fig. 1. $\Delta T$ images taken at $t=0.012 \mathrm{~s}$ during impact at $11.7 \mathrm{~J}$.
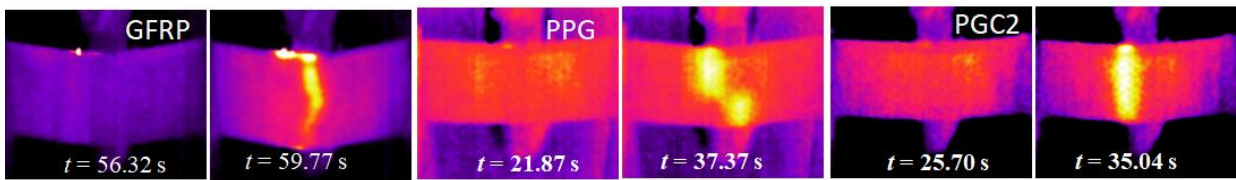

Fig. 2. Some thermal images of the three specimens under quasi-static bending.

\section{General comments}

The obtained results show that the visualization of thermal effects, due to the mechanical stresses, allows getting information, which can be exploited to better understand the materials reaction to applied forces and its damaging modes. In fact, monitoring the surface of a specimen while it is being pushed allows, through its surface temperature variations, to follow its deformation and bending at its critical curvature with formation of cracks and fibres breakage. In particular, under impact, the extension of the dark (cooled) zone accounts for the material deformation and for the overall impact-affected zone. The appearing of the first hot spot indicates damage initiation, while the warm area accounts for the whole delamination extension. Under quasi-static bending it is possible to see how the material bends to its critical curvature when it starts to break accompanied with formation of hot spots. The latter initially appear on the pushed surface, when the material starts to fail in compression, and later on the other surface, when it completely collapses. In other words, it is possible to see as dissimilar materials differently react to the applied force.

\section{REFERENCES}

[1] Liu Y, Farnsworth M, Tiwari A. A review of optimisation techniques used in the composite recycling area: Stateof-the-art and steps towards a research agenda J. Cleaner Production, 2017; 140: 1775-1781.

[2] Feraboli P, Kedward KT. A new composite structure impact performance assessment program, Compos. Sci. Technol. 2006; 66: 1336-1347.

[3] C. Meola, S. Boccardi, N.D. Boffa, F. Ricci, G. Simeoli, P. Russo, G.M. Carlomagno, New perspectives on impact damaging of thermoset- and thermoplastic-matrix composites from thermographic images, Comp. Struct. 152, 746-754, 2016.

[4] S. Boccardi, G.M. Carlomagno, C. Meola, P. Russo, G. Simeoli, Monitoring mechanical loading of polylactic acid matrix jute reinforced composites, Proc of XXIII AIMETA 2017, pp. 1934-42, ISBN: 978-889-42484-7-0. 